\title{
Application of an ultrasonic field for the removal of selected pesticides
}

\author{
Małgorzata Kida ${ }^{1, *}$, Sabina Ziembowicz ${ }^{1}$, and Piotr Koszelnik ${ }^{1}$ \\ ${ }^{1}$ Rzeszow University of Technology, Department of Chemistry and Environmental Engineering, \\ 35-959 Rzeszów, al. Powstańców Warszawy 6, Poland
}

\begin{abstract}
The work detailed in this study investigated the efficiency of oxidizing technologie for the removal of organochlorine pesticides (OCPs) from aqueous solutions. OCPs constitute an important group of environmental pollutants also known to be endocrine-disrupting compounds (EDCs). The particular focus has been on a process achieving oxidation through the use of ultrasonic waves, with the impacts of $\mathrm{pH}$, time on TOC and pesticide removal all being tested. Results confirm effective TOC decomposition using ultrasonic waves, albeit with reaction time showing a significant impact on results. Furthermore, results for TOC were not found to reflect those relating to the removal of individual substances from the tested mixture. Chromatographic analysis of reaction mixtures was therefore carried out after the selected processes had been applied, and an attempt to identify and characterize degradation products of the pesticides tested was also made. During the irradiation, the $\mathrm{H}_{2} \mathrm{O}_{2}$ concentration arising was also monitored. The results indicate that $\mathrm{H}_{2} \mathrm{O}_{2}$ is produced by cavitation during ultrasonic irradiation.
\end{abstract}

\section{Introduction}

While organochlorine pesticides (OCPs) are of great significance in pest control, they are also problematic due to the toxicity characterizing some kinds, and in particular the sheer volume applied every year. Much of that later finds its way into our water, air, and soil, with pesticides entering water via surface runoff or through leaching. Selected pesticides are listed by UNEP's Stockholm Convention, given their classification as persistent organic pollutants (POPs). Examples include HCB, aldrin, chlordane, DDT, endrin, heptachlor, and mirex. Because of the side effects of these biologically active components on the environment, most were eliminated from use in agriculture in the 1970s. A total ban was introduced in 1999, excluding DDT, whose application was allowed to continue until the end of 2010. Despite this, research continues to identify residues of these compounds and their metabolites in water and soils, and their migration in the environment leads to accumulation in aquatic systems and soils, as well as in food products $[1,2]$.

Several different means of destroying pesticides are applied commonly, while processes to achieve removal from aqueous solutions focus on sorption, coagulation, membrane processes, degradation by advanced oxidation techniques, and biodegradation. Today,

*Corresponding author: mkida@prz.edu.pl 
methods based on ultrasonic waves are considered promising, where water sources contaminated with compounds such as OCPs are concerned. Significant advantages of this technique compared to other advance oxidation processes (AOPs) are safety, cleanness, high penetrability in water medium, and the avoidance of secondary pollutant generation. This is a simple technique, requiring only electrical energy and easy to handle. However, literature reports on the removal of mixtures of these substances remain scarce, given the primary focus on the removal of single substances from model solutions. Where results are available, these indicate a need for process parameters to be selected separately for each type of substance. Furthermore, a key aspect of any process to achieve the oxidation of organic contaminants is the possibility that intermediate products (including some more toxic than the parent) may be formed [2-10].

The objective of the work detailed here was thus to investigate the efficiency of ultrasonic waves in removing OCPs from water samples, and to identify any by-products of decomposition. This research is only part of the research conducted by authors to remove these substances from water using AOPs. It is also possible to combine the ultrasonic field with other processes, which was shown in [11].

\section{Materials and methods}

\subsection{Reagents and materials}

A standard solution of mixed pesticides (Tetrachloro-m-xylene, $\alpha-\mathrm{HCH}, \gamma-\mathrm{HCH}$, Heptachlor, Aldrin, $\beta-\mathrm{HCH}$, Heptachlorepoxide Isomer $\mathrm{B}, \delta-\mathrm{HCH}$, Endosulfan I, $\gamma$-Chlordane, $\alpha$-Chlordane, 4,4'-DDE, Dieldrin, Endrin, 4,4'-DDD, Endosulfan II, 4,4'-DDT, Endrin Aldehyde, Endosulfan Sulfate, Methoxychlor, Endrin Ketone, Decachlorobiphenyl) was obtained from Supelco (Bellefonte, USA). Analytical grade n-hexane, methanol, and acetone for pesticide residue analysis were purchased from POCH (Gliwice, Poland). Ultra-pure water was obtained from Purix CNX-100. SPE cartridges and C18 sorbents were purchased from SiliCycle (Quebec City, Canada), $\mathrm{HCl}$ and $\mathrm{NaOH}$ solutions (to change the $\mathrm{pH}$ ) from $\mathrm{POCH}$. Reagent grade of potassium biphthalate, and potassium iodide (Chempur, Piekary Śląskie, Poland), as well as ammonium molybdate were obtained from POCH (Gliwice, Poland).

All glassware was submerged in $\mathrm{NaOH}$ solution for $24 \mathrm{~h}$, rinsed with ultra-pure water, and dried for $5 \mathrm{~h}$. The glassware was then rinsed with acetone, and heated at $280^{\circ} \mathrm{C}$ for $5 \mathrm{~h}$.

\subsection{Methods}

\subsubsection{Preparation of model solutions}

Model solutions were produced at concentrations of $20 \mu \mathrm{g} / \mathrm{L}$ for each pesticide, in ultrapure water, by adding a specific amount of a mixed standard solution of twenty-two OCPs, and mixing using an electromagnetic stirrer (Wigo). Concentrations of selected pesticides resembling those found in surface waters in Poland were achieved in this way.

\subsubsection{Use of ultrasonic waves}

The tested processes for removing of OCPs involved a Bandelin Sonopuls HD 3100 (Berlin, Germany), equipped with a $1.3 \mathrm{~cm}$-diameter titanium probe tip. The homogenizer operated at $20 \mathrm{kHz}$, with $200 \mathrm{~W}$ nominal power. Prepared samples $(500 \mathrm{ml}, 20 \mu \mathrm{g} / \mathrm{L}$ of each pesticide) were poured into the glass reactor. The temperature of the reaction medium was maintained at $25-30^{\circ} \mathrm{C}$, because at higher temperatures $\mathrm{H}_{2} \mathrm{O}_{2}$ is decomposed. Sonication was then conducted for durations of $5,15,30$, and $60 \mathrm{~min}$, ultrasonic intensity $3.2 \mathrm{~W} / \mathrm{cm}^{2}$ with the effects of $\mathrm{pH}$ and time on efficiency also being investigated. The ultrasonic process 
was terminated by neutralization of the sample, with ttal organic carbon (TOC) then being measured. TOC concentration was monitored with a TOC analyzer (Shimadzu). All batch experiments were performed in triplicate.

The concentration of $\mathrm{H}_{2} \mathrm{O}_{2}$ generated during sonication was determined using the iodometric method. Sample aliquots (volume: $2.0 \mathrm{~mL}$ ) from experiment were mixed in a quartz cuvette containing $0.75 \mathrm{~mL}$ of $0.10 \mathrm{M}$ potassium biphthalate and $0.75 \mathrm{~mL}$ of a solution containing $0.4 \mathrm{M}$ potassium iodide, $0.06 \mathrm{M}$ sodium hydroxide, and $10^{-4} \mathrm{M}$ ammonium molybdate. The mixed solution (total volume: $3.5 \mathrm{~mL}$ ) was allowed to stand for 2 minutes before absorbance was measured using a DR-5000 UV spectrophotometer.

\subsection{Determination of organochlorine pesticides using GC/MS}

To understand pathways to OCP oxidation, it was essential for degradation products formed from the pesticide mixture during the process to be identified. This is needed to evaluate in detail the formation of oxidation by-products and transformation products in oxidative treatment technologies reflectings potential toxicity.

\subsubsection{SPE - solid phase extraction}

Before samples were loaded, SPE cartridges were activated using $6 \mathrm{ml}$ of methanol, followed by $6 \mathrm{ml}$ of ultra-pure water. After activation, $100 \mathrm{ml}$ aqueous samples were passed through the SPE cartridges, which were then dried under vacuum for $20 \mathrm{~min}$ to remove retained water. Analytes were then eluted with $20 \mathrm{ml}$ solutions of n-hexane and acetone $(9: 1, \mathrm{v} / \mathrm{v})$, prior to samples being evaporated using a rotary evaporator (Heidolph, Germany). The analytes were then redissolved in $1 \mathrm{ml}$ methanol.

\subsubsection{Chromatographic analysis of OCPS}

Analysis of the pesticides was performed using Trace GC ULTRA with TriPlus coupled to an ITQ 1100 (Thermo Scientific). A ZB-5MSplus Zebron Capillary GC Column (analytical column) of $30 \mathrm{~m} \times 0.25 \mathrm{~mm} \times 0.25 \mu \mathrm{m}$ was used. The temperature program was as follows: initial temperature, $100^{\circ} \mathrm{C}$ held for $1 \mathrm{~min}$, increasing temperature ramp by $20^{\circ} \mathrm{C} / \mathrm{min}$ to $210^{\circ} \mathrm{C}$ and then held for $1 \mathrm{~min}$, increasing temperature ramp by $5^{\circ} \mathrm{C} / \mathrm{min}$ to $300^{\circ} \mathrm{C}$ and then held for $2 \mathrm{~min}$; total time $27.5 \mathrm{~min}$. Helium was used as the carrier gas. MS parameters: ion source $-230^{\circ} \mathrm{C}$, transfer line temperature $-280^{\circ} \mathrm{C}$, full scan, mass range $50-650$. Recoveries for pesticides resulted in values in the range $81-127 \%$.

\section{Results}

The investigation addressed the possibility of TOC being removed from water by way of a process involving ultrasonic waves. Experiments involved irradiation times in the 5 to $60 \mathrm{~min}$ range, prior to the collection and analysis of samples. The effects of the ultrasonic process at various $\mathrm{pH}$ levels and after different time intervals are as shown in Fig. 1. The efficiency of destruction pesticides achieved at $\mathrm{pH}$ levels of 3,7 , and 11 were $68.2 \% 56.4 \%$ and $49.3 \%$ respectively. Other parameters were: $20 \mu \mathrm{g} / \mathrm{L}$ concentration for each pesticide, run duration $5 \mathrm{~min}$, frequency $20 \mathrm{kHz}$, and ultrasonic intensity $3.2 \mathrm{~W} / \mathrm{cm}^{2}$. These results demonstrate that ultrasonic waves were more efficient at removing pesticides at $\mathrm{pH} 3$, as opposed to $\mathrm{pH} 7$ or 11 .

Continuation of the process was found to be associated with a rise in solution temperature that related directly to the amount of energy consumed. Specifically, after 5 , 15,30 , and $60 \mathrm{~min}$ respectively, some $11.3,28.4,39.8$, and $77.6 \mathrm{~kJ}$ had been introduced. Notwithstanding such changes, $\mathrm{pH}$ values of the solution remained stable. 


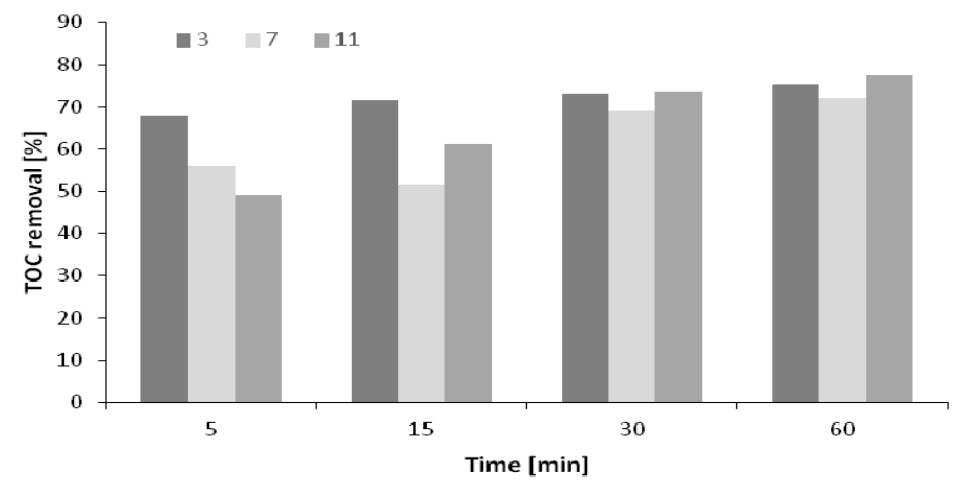

Fig. 1. Effect of sonication time on the removal of TOC.

When an aqueous solution is irradiated ultrasonically hydrogen peroxide is produced. Hydrogen peroxide is involved in AOPs and account for the relationship between ultrasonic action and AOPs. The influence of initial $\mathrm{pH}(3,7,11)$ on the production of hydrogen peroxide in deionized water without pollutant is as shown in Fig. 2.

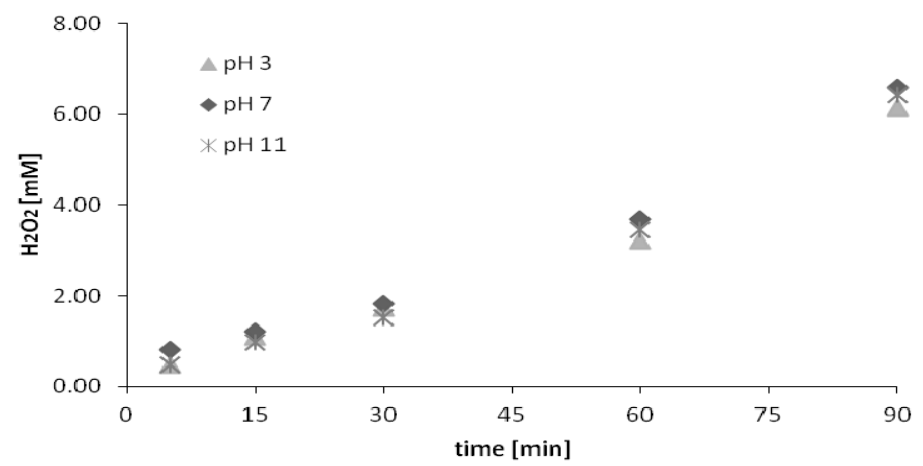

Fig. 2. Influence of $\mathrm{pH}$ values and duration of ultrasonic irradiation (at a constant intensity of $3.2 \mathrm{~W} / \mathrm{cm}^{2}$ ) on the formation of $\mathrm{H}_{2} \mathrm{O}_{2}$.

The application of GC/MS allowed for determinations of the studied pesticides using analytical standards, and of some of the oxidation by-products using the NIST identification library. GC-MS analyses of the solutions were run before and after processing (oxidation by ultrasonic waves). The time selected for the GC/MS analysis was $60 \mathrm{~min}$.

Oxidation using ultrasonic waves did not achieve complete removal of pesticides such as $\alpha-\mathrm{HCH}, \gamma-\mathrm{HCH}$, and Heptachlor. Following the process, $\alpha-\mathrm{HCH}$ remained detectable at $3.3 \mu \mathrm{g} / \mathrm{L}$, $\gamma-\mathrm{HCH}$ at $4.2 \mu \mathrm{g} / \mathrm{L}$, and Heptachlor at $3.8 \mu \mathrm{g} / \mathrm{L}$. Other pesticides have been totally removed.

\section{Discussion}

This work detailed here achieved organochlorine pesticides degraded through a process deploying ultrasonic waves. Specifically, about $50-80 \%$ of the initial concentration of TOC was degraded. Barbusinski et al. [12] investigated the degradation of pesticides using Fenton's reagent and hydrogen peroxide. In that case, organochlorine pesticides were destroyed with an efficiency of more than $90 \%$, for individual pesticides (DDT, $\alpha-\mathrm{HCH}$, $\beta-\mathrm{HCH}, \gamma-\mathrm{HCH}$ ), with a dose of $\mathrm{H}_{2} \mathrm{O}_{2}=2.5 \mathrm{~g} / \mathrm{L}$ proving sufficient in most cases, and for example degrading more than $90 \%$ of $\gamma-\mathrm{HCH}$. It is worth adding that a higher dose was not found to cause any improvement in those experiments. 
The work described here examined the effect of sonication time on the removal of OCPs, revealing that the percentage of degradation increased with time. However, since no significant change occurred beyond $30 \mathrm{~min}$, this time was selected as optimal. Similar results have also been obtained for the sonochemical degradation of other organic compounds $[13,14,15]$. A number of studies also report that initial pesticide concentrations decrease with sonication time up to a certain point, beyond which a steady state phase is achieved. It is possible that OCPs do not degrade directly to water and carbon dioxide, but reacted via a series of intermediates, in a process that needed some optimal time to achieve degradation.

The process by which organochlorine pesticides decompose can be described by considering the effectiveness of ultrasonic irradiation in forming hydroxyl radicals. These react with pesticides by addition to double bonds or by hydrogen abstraction. Hydroxyl radicals are produced in the hot vapor phase, and may either react there, or diffuse into and react within the surrounding liquid phase. Sonolysis of water also produces hydrogen peroxide. Across the $\mathrm{pH}$ range studied, the rate of formation of $\mathrm{H}_{2} \mathrm{O}_{2}$ was independent of the initial value. However, the $\mathrm{pH}$ values of solutions are shown to influence the sonochemical degradation of organic pollutants markedly. The influence of the $\mathrm{pH}$ of solutions is probably therefore due to the chemical structure and properties of the substance involved [16].

GC-MS analyses were carried out on solutions before and after processing. Oxidation by ultrasonic waves does not achieve full removal of pesticides like $\alpha-\mathrm{HCH}, \gamma-\mathrm{HCH}$ and Heptachlor, but these pesticides are in general known to be resistant to methods of oxidation applied. They are substances very resistant to environmental influences, such as temperature, light, and oxidation. Heptachlor is a chlorinated dicyclopentadiene, classified as one of the so-called POPs restricted by the Stockholm Convention in 2001. While the half-life of heptachlor is 250 days, traces of this pesticide prove detectable in soil even 14 and 16 years after application. A widespread reaction in the environment is heptachlor epoxidation to the more persistent heptachlor epoxide [17,18]. $\gamma-\mathrm{HCH}$, known commercially as Lindane, is a highly chlorinated, recalcitrant pesticide that has been targeted for remediation given both its toxicity and its persistence [19].

Combined chromatogram and GC/MS spectra results suggest no formation of byproducts during the oxidation process - a result that confirms research conducted by Haag et al. [20], Blanco et al. [21], and Barbusinski et al. [12]. Data in the literature on the degradation of selected pesticides allow it to be assumed that no stable toxic intermediate products are formed in oxidation reactions with $\mathrm{OH}^{-}$radicals. Generally, pesticides are oxidized to $\mathrm{CO}_{2}$. However, carboxylate anions, such as formates, acetates, or oxalates, are formed as intermediate, non-toxic products. Pesticides containing halogens, sulfur, or phosphorous heteroatoms are oxidized quantitatively to form chlorides, sulfates, and phosphates. Pesticide molecules are transformed into primary oxidation products, which often undergo either spontaneous transformation (e.g. protonation, dimerization, isomerization, and tautomerization) or further oxidation by another hydroxyl radical [13,22].

The introduction of pesticides into the sewer system or the environment needs to follow treatment and purification makes use of physical, chemical, physico-chemical and biological methods. As technology for the removal of organic contaminants from water develops, heed should be taken not only of effectiveness, but also of efficiency (with reference made to the costs of the process), and the likely formation of toxic by-products. A key advantage of the process described here is that no formation of by-products has been noted. In most cases, the goal of current technical applications of advanced oxidation processes is the destruction of pollutants in water, rather than the energy-intensive complete degradation of the content in the substrate. The most important economic parameter is thus the efficiency of substrate consumption [21-27]. 


\section{Conclusion}

- The studies conducted show how the concentrations of pesticides in solution may be reduced through treatment processes involving ultrasonic waves. These prove effective in destroying the OCPs in water.

- Sonodegradation of pesticides was achieved successfully with various sonication times and at different $\mathrm{pH}$ levels.

- $\mathrm{H}_{2} \mathrm{O}_{2}$ can be generated by cavitation in an aqueous solution. Initial $\mathrm{pH}$ did not affect the efficiency of $\mathrm{H}_{2} \mathrm{O}_{2}$ formation substantially.

\section{References}

1. J. Kaleta, JCEEA 38 (2004)

2. J. Kaleta, M. Kida, P. Koszelnik, D. Papciak, A. Puszkarewicz, B. Tchórzewska-Cieślak, Arch. Environ. Prot. 43 (2017)

3. M. Smol, M. Włodarczyk-Makuła, K. Mielczarek, J. Bohdziewicz, D. Włóka, Polycycl. Aromat. Comp. 36 (2016)

4. A. Rosińska, L. Dąbrowska, Desali. Water Treat. 51(7-9) (2013)

5. M. Włodarczyk-Makuła, LAB Lab., Aparat., Bad. 20 (2015)

6. M. Włodarczyk-Makuła, Physical and chemical fates of organic micropollutants, Scholars' Press 2015.

7. M. Matouq, Z. Al-Anber, N. Susumu, T. Tagawa, H. Karapanagioti, Sep. Purif. Technol. 135 (2014)

8. A. Żarczyński, A. Stopczyk, M. Zaborowski, Z. Gorzka, M. Kaźmierczak, Ochr. Środ. 32 (2010)

9. E. Kusvuran, E. Oktay, J. Hazard. Mater. 106 (2004)

10. E. Nikfar, M.H. Dehghani, A.H. Mahvi, N. Rastkari, M. Asif, I. Tyagi, S. Agarwal, V.K. Gupta, J. Mol. Liq. 213 (2016)

11. M. Kida, S. Ziembowicz, P. Koszelnik, Sep. Purif. Technol. 192 (2018)

12. K. Barbusiński, K. Filipek, Pol. J. Environ. Stud. 10(4) (2001)

13. K. Ikehata, M.G. El-Din, J. Environ. Eng. Sci. 5(2) (2006)

14. C. Wu, X. Liu, D. Wei, J. Fan, L. Wang, Water Res. 35 (2001)

15. Y. Jiang, C. Petrier, T.D. Waite, Ultrason. Sonochem. 9 (2002)

16. M.H. Dehghani, A. Fadaei, Environ. Prot. Eng. 39 (2013)

17. World Health Organization, Heptachlor, Concise International Chemical Assessment Document 70 (2006), Geneva, Switzerland.

18. P. Xiao, T. Mori, I. Kamei, R. Kondo, FEMS microbiology letters 314 (2011)

19. T.M. Phillips, A.G. Seech, H. Lee, J.T. Trevors, Biodegradation 16(4) (2005)

20. W.R. Haag, C.C.D. Yao, Environ. Sci. Technol. 26(5) (1992)

21. J. Blanco, S. Malato, B. Milow, M.I. Maldonado, H. Fallmann, T. Krutzler, R. Bauer, J. Phys. IV, 9(P3) (1999).

22. J. Naumczyk, P. Marcinowski, J. Bogacki, Environ. Protec. Eng. 43(2) (2017)

23. K. Pochwat, D. Słyś, S. Kordana, J. of Hydr. 549 (2017)

24. J. Kaleta, A. Puszkarewicz, D. Papciak, Environ. Protec. Eng. 33(3) (2007)

25. K. Pochwat, E3S Web Conf. Volume 17 (2017)

26. K. Ignatowicz-Owsieniuk, I. Skoczko, Pol. J. Environ. Stud. 11(4) (2002)

27. K. Jóźwiakowski, M. Marzec, J. Fiedurek, A. Kamińska, M. Gajewska, E. Wojciechowska, A. Kowlaczyk-Juśko, Sep. Purif. Technol. 173 (2017) 